\title{
A iniciativa Open Access no acesso à informação técnico-científica nas Ciências da Saúde
}

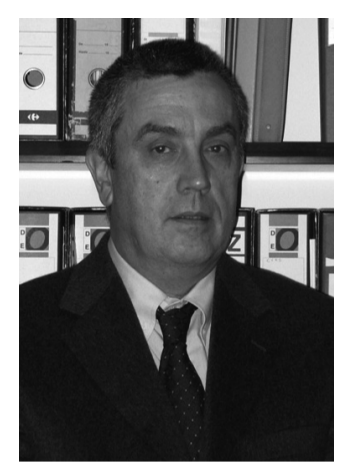

\section{Jawier Sanz} Valero

Universidad de Alicante, Departamento de Enfermagem Comunitária, Medicina Preventiva e Saúde Pública e História da Ciência, Alicante, Espanha j.sanz.000@recol.es

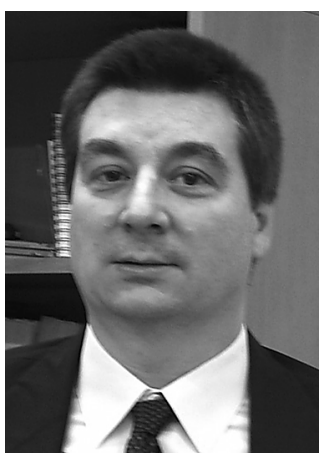

\section{Jorge Veiga} de Cabo

Escuela Nacional de Medicina del Trabajo, Instituto de Salud Carlos III, Madrid, Espanha jorge.veiga@isciii.es

\section{Luis David Castiel}

Fundação Oswaldo Cruz, Departamento de Epidemiologia e Métodos Quantitativos em Saúde, Escola Nacional de Saúde Pública, Rio de Janeiro, Brasil

luis.castiel@ensp.fiocruz.br

\section{Resumo}

A iniciativa Open Access (OAI) trata de documentos publicados eletronicamente, permitindo acesso livre, direto, permanente e gratuito ao texto completo da literatura científica e de pesquisa pela internet. Isto pressupõe o livre aproveitamento da informação científica, sempre que seja considerado o reconhecimento da autoria. As três principais manifestações sobre OAI estão contidas nas declarações de Budapeste (2002), de Bethesda (2003) e de Berlim (2003). O Open Access é compatível com o copyright, o copyleft, a revisão por pares, a impressão, a preservação e outras características associadas à literatura acadêmica convencional. A diferença de maior relevância consiste no fato de que o acesso à informação não é pago pelos leitores e, portanto, não existem barreiras à pesquisa. Mesmo assim, o êxito da OAI não depende somente da disponibilidade dos documentos, mas no apoio da comunidade científica.

\section{Palavras-chave}

Acesso aberto, acesso à informação, armazenamento e recuperação da informação, internet, documentação

\section{Introdução}

$\mathrm{O}$ acesso aberto à literatura científica, Open Access Iniciative (OAI), tem cada vez mais seguidores. Para isto acontecer, contribuíram as facilidades técnicas atuais. De fato, muitos teóricos denominam a época atual como a sociedade da informação ou sociedade digital. A razão para denominá-las assim reside na influência do paradig- ma tecnológico dominante, que ocasionou mudanças nas relações humanas e nas formas de viver. Outros, de forma mais pragmática, a denominaram "era pós-Gutenberg" (HARNARD, 1991).

Portanto, não é de se estranhar, dado o atual crescimento da internet, que se utilizem esses recursos para ajudar as instituições acadêmicas e os pesquisadores 
particulares na difusão da comunicação científica (VASCONCELLOS-SILVA et al., 2003), promovendo os intercâmbios e a visibilidade de sua produção científica, favorecendo assim a construção e o desenvolvimento do conhecimento (LIMA-LEITE, 2006; SANS-VALERO, 2006). Mas, deve-se prestar atenção aos efeitos colaterais que podem limitar as oportunidades de acesso (PERINE, 2007), e levar em conta que, cada vez mais, é necessária a vigilância da qualidade da informação obtida na internet, assim como a proteção do usuário, o direito à informação e a proteção dos dados.

Apesar do consenso sobre as vantagens do OAI entre os acadêmicos e a concordância de que este seria o modo ótimo para a distribuição dos resultados das pesquisas com financiamentos públicos, ainda constitui um fenômeno minoritário no mundo da edição, ainda que, felizmente, seus partidários estejam em números cada vez maiores. As possibilidades oferecidas pelas novas tecnologias para as publicações científicas se vêem restringidas pelas barreiras, sobretudo econômicas, que são impostas pelas grandes empresas editoriais. Os principais problemas são: o abusivo aumento dos preços das assinaturas e a imposição de contratos "por pacotes" (big deals) (MELERO, 2005; CANCELA-RODRÍGUEZ, 2003). Ainda que, convenhamos, esse encarecimento tenha gerado um apoio maior à OAI.

O objetivo e a finalidade do presente trabalho é proporcionar uma visão abrangente sobre a situação atual da iniciativa Open Access, analisando alguns aspectos relacionados ao copyright e ao copyleft, e informando acerca dos principais acessos à literatura científica através dos portais que assumiram essa iniciativa.

\section{Open Access: uma iniciativa de divulgação do conhecimento}

Um passo importante na visualização de documentos é o surgimento do formato PDF (Portable Document Format) como padrão de fato na distribuição de documentos eletrônicos em todo o mundo. O formato PDF consiste em um arquivo universal que preserva todas as fontes, a aparência, as cores e os gráficos de qualquer documento-fonte, sem que importe nem a aplicação nem a plataforma usadas em sua criação. Os arquivos PDF são compactos e podem ser compartilhados, visualizados, navegados e impressos exatamente como pretenda e queira seu gerador.

O Open Access em revistas de acesso aberto trata de publicações que passaram por mecanismos de avaliação e que se encontram publicadas eletronicamente. Isto permite um acesso livre, direto, permanente e gratuito ao texto completo da literatura científica e de pesquisas através da internet, ainda que a maioria delas não tenha deixado de ser cópia em fac-símile de seu produto em papel.

Pode também se tratar de outros recursos documentais diferentes em relação a revistas, como livros, teses, manuais de prática clínica ou e-prints (JAEN CASQUERA, 2004). Os e-prints, segundo a Budapest Open Access Initiative (BOAI), organismo pioneiro no acesso livre, são documentos digitais cuja intenção é a publicação, mas que podem ou não ter passado por uma revisão por pares. Quando o documento não passou pela revisão por pares, se denomina preprint, e quando houve tal revisão e está pronto para sua publicação, chama-se postprint. Os preprints e os postprints compõem os eprints (EPRINTS ORG., 2007).

É claro que, na opinião de alguns autores, é mais importante a qualidade da revista que o acesso aberto na hora de se escolher onde publicar os trabalhos (SCHROTER, 2005), mas também é fonte de preocupação sobre quem vai controlar, e como, a qualidade do que se publica. Os autores desejam que seus trabalhos sejam publicados com a maior rapidez possível em locais de evidente qualidade e que apresentem alta visibilidade. No que se refere à entrega de manuscritos, os editores da grande maioria das revistas, para não dizer a totalidade, aceitam a submissão dos originais por via eletrônica (CANEDO ANDALIA, 2002).

Inclusive, desde o ponto de vista da maior evidência científica, o fato de se facilitar o conhecimento dessa documentação não deveria gerar discussão. Os patrocinadores e as agências financiadoras não desejam esbanjar recursos levando a cabo pesquisas já realizadas. Ao mesmo tempo, pesquisadores que estão desenvolvendo meta-análises ou revisões sistemáticas necessitam poder identificar tudo o que foi realizado sobre esse tema concreto, com a finalidade de se evitar o viés de publicação (HORTON, 1999).

As três principais manifestações sobre o acesso aberto estão contidas nas declarações de Budapest, (fevereiro/2002, www.soros.org/openaccess/read.shtml), de Bethesda (junho/2003, www.wsis-si.org/mdpi-bethesda. pdf) e de Berlim (outubro/2003, www.zim.mpg.de/openaccess-berlin/berlindeclaration.html).

Partia-se da convergência entre a vontade dos cientistas de publicar e difundir os resultados de seus trabalhos e a disponibilidade cada vez maior de publicações científicas na rede.

O compromisso para o estabelecimento do acesso aberto como um recurso plausível deve satisfazer duas condições (SOCIEDAD MAX PLANCK, 2003) (Declaração de Berlim sobre acesso aberto):

1. $\mathrm{O}(\mathrm{s})$ autor(es) e o depositário da propriedade intelectual devem garantir o direito irrevogável e mundial de acessar um trabalho erudito, o mesmo que licencia para copiá-lo, usá-lo, distribuí-lo, transmiti-lo e exibi-lo publicamente, e para fazer e distribuir trabalhos derivativos, em qualquer meio digital para qualquer propósito responsável, tudo sujeito ao reconhecimento apropriado de autoria (os padrões da comunidade continuarão provendo os mecanismos para que sejam satisfeitos o reconhecimento apropriado e uso responsável das obras publicadas, como agora se faz), o mesmo que o direito de fazer cópias impressas em pequeno número para seu uso pessoal.

2. Uma versão completa do trabalho e de todos os seus materiais complementares (que inclui uma cópia da permissão mencionada no item anterior) sob um con- 
veniente formato eletrônico padrão deve ser depositada (e assim é publicada) em pelo menos um repositório online que utilize padrões técnicos aceitáveis (tais como as definições do Acesso Aberto), que seja apoiado e mantido por uma instituição acadêmica, sociedade erudita, agência governamental ou uma organização bem estabelecida que procure implementar o acesso aberto, distribuição irrestrita, interoperabilidade e capacidade de arquivamento no longo prazo.

O Open Access é compatível com o copyright, o copyleft, a revisão por pares, a impressão, a preservação e outras características associadas à literatura acadêmica convencional. A diferença substancial consiste no fato de que o acesso à informação não é pago pelos leitores e, portanto, não existem barreiras à pesquisa.

As principais vantagens do Open Access seriam (OPEN ACCESS CHARTER, 2007: REASONS TO PUBLISH, 2007):

- Acesso à leitura e à pesquisa de forma gratuita.

- Na maioria das vezes, não é necessário transferir o direito de cópia.

- Rápida revisão pelos pares.

- Publicação imediata.

- O envio do documento de forma eletrônica.

- Maior visibilidade e promoção do trabalho.

- Informação mais atualizada.

O êxito da iniciativa Open Access não se localiza apenas na disponibilidade e no acesso aos documentos científicos, mas no progressivo apoio da comunidade científica e de suas instituições.

Dando importância à recomendação da Dra. Melero, para aprofundar o entendimento da iniciativa Open Access, seria recomendável a leitura dos trabalhos publicados por ela mesma (MELERO, 2005), aqueles elaborados periodicamente por Peter Súber, Timeline of the free online scholarship movement (SUBER, 2007), e a monografia escrita pelo Prof. Bailey, Open access bibliography (BAILEY, 2005), onde se recolheram cerca de 1.300 referências classificadas por temas e publicadas no período entre 1999 a 2004.

Para que as revistas científicas cumpram a missão de comunicar o conhecimento científico que nelas se publica, devem ter a mais ampla difusão possível.

A difusão direta é aquela que se obtém em função do número de exemplares que são editados, que, por sua vez, está condicionada pela quantidade de assinaturas contratadas. Mas é preciso levar em consideração que nem todas as assinaturas têm o mesmo valor. Para efeitos da difusão do conteúdo de uma revista, não é a mesma coisa uma subscrição pessoal em relação a de um departamento de pesquisa de uma universidade, ou a de uma biblioteca acadêmica. A difusão indireta é o que alcançam as publicações através da inclusão dos resumos ou de seus artigos em bases de dados e outras fontes de referência que são utilizadas para a pesquisa de informação. As revistas incluídas nas mais importantes bases de dados temáticas obtêm uma difusão e visibilidade internacional muito maior do que as que não estão recolhidas nessas fontes secundárias de recuperação de informação (VÁZQUEZ VALERO, 2003).

É evidente que a Internet ajuda na divulgação de conhecimentos em escala mundial. Isto quer dizer que a presença de uma revista na Internet facilita a sua difusão planetária, uma vez que pode ser examinada por uma enorme população de potenciais usuários. Por esta razão, para uma revista, a possibilidade de estar presente na Internet é um modo seguro de tornar-se visível para grande parte da comunidade científica, visibilidade que aumenta caso seja publicada em inglês (VEIGA DE CABO, 2001). Esta visibilidade será ainda maior se a informação colocada na rede for completa e armazenada em revistas de qualidade. A exigência de avaliação prévia do trabalho antes de sua inclusão na rede será também conclusiva para calibrar a qualidade.

As vantagens que a Internet oferece têm sido determinantes no momento em que o papel deixe de ser imprescindível como suporte da transmissão do conhecimento. Essas qualidades fazem com que surjam, dentro da mesma comunidade científica, correntes dirigidas a facilitar o livre acesso à literatura especializada. Duas das principais alternativas são: o auto-arquivamento (self-archiving) e as revistas com acesso aberto (open access) (VEIGA DE CABO, 2001).

No auto-arquivamento, são os autores que armazenam seus trabalhos, seja situando-os em coleções temáticas (exemplo: o E-LIS, comentado mais adiante), seja situando-os em coleções institucionais, algo cada vez mais promovido pelas universidades. O auto-arquivamento já é um movimento internacional com rápido desenvolvimento, que virá a se concretizar em locais de armazenamento para receber a produção científica. Podem ser considerados uma espécie de biblioteca virtual, mas devido à multiplicidade da produção e, portanto, à dificuldade de encontrá-los, o surgimento de coleções temáticas será bem-vindo, e, como se mencionou, o ELIS é um magnífico exemplo.

Quanto às revistas com acesso aberto, podemos distinguir:

a) revistas editadas somente em formato eletrônico. Como exemplo, podemos citar aquelas contidas na BioMed Central ou na PLoS Medicine;

b) revistas editadas em ambos os formatos, papel e eletrônico. Entre elas estariam aquelas colecionadas em SciELO ou em DOAJ;

c) revistas em ambos os formatos que permitem o acesso à edição online depois de um período de tempo. Neste grupo estariam aquelas incluídas na PubMed Central e outras que são acolhidas a esta modalidade, como New England Journal of Medicine ou Lancet.

No caso de aceitarmos que a literatura científica seja acessível através da internet sem custos para os leitores, não devemos esquecer que a produção, armazenamento e difusão sem custos não são possíveis.

Hoje em dia, um número cada vez maior de revistas permite o acesso aberto ao artigo, mas cobra uma taxa a seu autor. Ou seja, os custos de manutenção, revisão e edição recaem sobre os autores ou sobre as instituições 
às quais pertençam. O modelo de publicação baseado no fato de que ou o autor ou a instituição venha a manter os gastos editoriais apresenta algumas dúvidas, preocupação e desconfiança sobre sua sustentabilidade (MUÑOZ TINOCO, 2005).

Portanto, existem duas estratégias para favorecer a publicação em acesso aberto:

1. publicar em uma revista de acesso aberto. Denomina-se Gold Open Access;

2. publicar em qualquer tipo de revista de acesso restrito e, posteriormente, depositar o trabalho em uma coleção institucional. Denomina-se Green Open Access.

\section{Impacto versus Visibilidade}

A visibilidade de um trabalho é um fator determinante no processo de citação. Um trabalho que não se encontra visível, acessível e disponível não pode ser considerado, avaliado ou utilizado na realização de outros trabalhos. Neste sentido, uma variável importante é a possibilidade de ter-se acesso livremente ao texto completo da contribuição. Mais ainda: constitui-se um fator decisivo.

Até pouco tempo atrás, o principal indicador para medir a trilha que uma publicação deixava dentro do coletivo científico era, e infelizmente continua sendo, o índice de impacto (contabilidade das citações sobre uma publicação de acordo com os critérios emanados do Institute for Scientific Information). Na verdade, o fator de impacto de uma revista não é estatisticamente representativo do índice de citação de seus artigos.

O aparecimento da iniciativa Open Access, juntamente com as possibilidades telemáticas, permite novas modalidades de se conhecer a visibilidade ou o interesse que suscita cada documento em si mesmo.

Entre estes diferentes indicadores temos:

- Hits: número de vezes em que se acessa ao documento.

- Downloads: número de vezes que se baixa o documento.

- Visibilidade: inclusão a partir do lugar de onde se localiza o documento, com vínculo (link) a outra página da Web.

Em geral, a publicação disponível na rede aumenta a sua consulta. Assim, os estudos bibliométricos demonstram um incremento na referência dos documentos de livre acesso depositados na rede em relação aos que não estão ou são de acesso restrito (HARNARD, 2007; ANTELMAN, 2004), apresentando diferenças significativas a favor das revistas de acesso aberto quando se estudava o índice de imediatismo (documentos com uma idade igual ou inferior a 1 ano). Ou seja, maior rapidez na difusão da documentação científica (estudo realizado em 2004 pelo Institute for Scientific Information (ISI) com os dados do Journal Citation Report de 2003) (THONSOM CORPORATION, 2004).

Ainda não existe um modelo de análise totalmente aceito e que consiga suprir as características do modelo do ISI, ainda que coexistam algumas propostas como as anteriormente assinaladas no entorno da edição eletrônica. Da mesma forma, podemos assinalar os trabalhos realizados pelo Open Citation Project (OPCIT, 2007), cujo fim é o de estudar e de construir ferramentas para a análise de citações nas publicações eletrônicas de acesso aberto. Entre elas, podemos apontar a CITEBASE (www. citebase.org).

Quando se deposita um documento científico em um repositório, a difusão e o uso eficiente do mesmo precisam estar claramente identificados e quantificados, e devem ser, obrigatoriamente, mensuráveis.

Além disto, a declaração de Bethesda recolhe o compromisso das instituições signatárias para respaldar e fomentar entre os pesquisadores o uso de revistas que contemplem esse conceito. Ainda assim, se comprometem a desenvolver novos métodos de avaliação e de reconhecimento dos méritos acadêmicos dos cientistas que publiquem sob a chancela dessa iniciativa.

\section{A Propriedade Intelectual: Open Access Movement}

As facilidades que a telemática oferece para a difusão e para a acessibilidade à comunicação e à documentação científica contrastam com as barreiras econômicas e dos direitos de reprodução (copyright) impostas pelas grandes empresas editoriais que controlam a maioria do mercado das publicações científicas. Como resposta a essa situação, surge a reação dos pesquisadores e dos gestores da informação acadêmica, dando origem ao que se denominou "movimento para o livre acesso (open access movement) às publicações científicas” (MELERO, 2004). A Universidade de Harvard publicou, inclusive, em seu periódico, os aspectos orçamentários correspondentes à suposta garantia de acesso à literatura científica de todos os usuários de suas bibliotecas (LIBRARIES, 2004).

O conceito open access não tem relação apenas com a acessibilidade à documentação científica, mas com a idéia de eliminar a obrigatoriedade de cessão do copyright dos artigos publicados, o que facilita ao autor seu depósito em arquivos e repositórios institucionais ou temáticos.

O copyright é um conjunto de direitos atribuídos automaticamente ao autor (autores) pela legislação da maioria dos países. É uma combinação de direitos éticos e direitos de exploração. Os éticos costumam estar bem presentes no mundo acadêmico e não costumam ser questionados e nem entram em debate (permitam-nos esta licença), coisa bem distinta se falamos dos direitos de exploração. Hoorn e van der Graaf elaboraram um bom trabalho sobre as atitudes dos autores do Reino Unido e dos Países Baixos ante os direitos de autoria cuja leitura é aconselhável (HOORN, 2006).

Na questão entre o acesso aberto e o restrito, estamos lidando com algo que não deixa de ser uma discussão em torno ao desenvolvimento e da defesa da propriedade intelectual e, por extensão, do direito dos autores exercerem o controle e a vigilância sobre suas obras. A propriedade intelectual de uma obra literária, artística ou científica pertence ao autor pelo simples fato de sua criação. Neste sentido, é melhor não supor que os autores 
têm a obrigação de comunicar seus trabalhos de forma gratuita. Seria presumir que os direitos de exploração da obra são do autor, enquanto não existe um contrato devidamente formalizado por ele que diga o contrário (VIVEZ GRÁCIA, 2005). Portanto, toda cessão de direitos deverá formalizar-se por escrito. Não obstante, atualmente, a maioria das revistas permita aos autores o auto-arquivamento de uma cópia de seu trabalho, não do artigo publicado. Ou seja, a versão auto-arquivada pelo autor não é o substituto da versão oficial do editor da revista onde o artigo foi publicado. (Para consultar as normas da maioria das principais revistas científicas acessar a página da SHERPA - www.sherpa.ac.uk - da Universidade de Nottingham).

Em 2004, algumas instituições, como, por exemplo, o US National Institute of Health ou a fundação Wellcome Trust, decidiram que as publicações derivadas de projetos financiados com seus recursos deveriam ser armazenadas em bases de dados ou repositórios de livre acesso. Estas políticas institucionais a favor do acesso aberto desencadearam uma discussão sobre o patrocínio de estratégias sobre o armazenamento da produção científica criadas com fundos públicos. Neste sentido, existe uma corrente denominada "arquivangelista", cujo máximo representante é o professor da Universidade de Southampton (UK), Stevan Harnard, que defende que a única maneira de se conseguir um grande apoio à iniciativa open access é obrigando os autores a guardarem uma cópia de seu trabalho nos arquivos de sua instituição.

Então, deve ficar patente que o armazenamento de um trabalho em formato aberto não implica a renúncia do autor a seus direitos. Este pode explorá-lo da maneira que lhe pareça a mais conveniente (ANDERSON, 2004). Pôr à disposição uma obra em acesso aberto não significa, em nenhum dos casos, a abdicação, por parte do autor, de seus direitos.

Neste sentido, seria interessante destacar o crescente apoio ao movimento copyleft, entendendo como tal o fato de se promover um maior controle dos criadores sobre suas obras, pesquisas e projetos, e uma remuneração compensatória mais razoável por seu trabalho. Permite também aos usuários finais melhor acesso e proveito dos bens desses tipos de licenças não restritivas. Isto é, o autor detém o poder de decidir como e em que condições vai reproduzir e distribuir sua obra. Geralmente são permitidas três circunstâncias: cópia e distribuição não comercial (o mínimo exigível para que uma obra seja considerada copyleft), obras derivadas e distribuição comercial; tudo isto sem a necessidade de novas permissões do autor.

Por outro lado, deve-se levar em conta que todos os princípios éticos recolhidos sobre a publicação científica são aplicáveis à edição Open Access, e que talvez sobre este tema tenhamos que abrir um amplo debate.

\section{Portais vinculados ao Open Access}

Entre as plataformas Open Access já plenamente consolidadas na Internet, que contam com um reconhecido prestígio e facilitam o acesso livre e gratuito e permanente de texto completo, poderiam ser citadas:
- Access to Global Online Research in Agriculture - AGORA (www.aginternetwork.org/es): desenvolvido pela Organização das Nações Unidas para a Agricultura e a Alimentação (FAO) junto a patrocinadores privados, dá acesso de modo destacado a coleções bibliográficas digitais nos campos da alimentação, agricultura, ciências do meio ambiente e ciências sociais. Dá acesso a 918 publicações para instituições em 107 países em vias de desenvolvimento.

- Bioline International (www.bioline.org.br): serviço de acesso aberto a publicações científicas, revisadas por pares, produzidas em países em vias de desenvolvimento cujo objetivo é facilitar um acesso sustentável e promover o aumento da qualidade. Os principais responsáveis são o Centro de Referência em Informação Ambiental (CRIA), Open Society Institute e a Universidade de Toronto.

- BioMed Central (www.biomedcentral.com): casa editorial independente, do Reino Unido, orientada para proporcionar o acesso livre e imediato à pesquisa biomédica de qualidade e revisada por pares. Permite o acesso a mais de 140 revistas sobre ciências da saúde.

- Directory of Open Access Journals - DOAJ (www.doaj. org): concebido pela Lund University Libraries da Suécia. Diretório de revistas de livre acesso, dispõe de textos completos que cobrem publicações de âmbito científico e acadêmico, controlando a qualidade do que é publicado. Atualmente contém 2.009 revistas.

- Documents in Information Science - DoIS (wotan. liu.edu/dois): serviço para localizar e baixar documentos de pesquisa sobre o campo das ciências da informação. É uma base de dados de artigos e conferências publicadas em formato eletrônico na área da biblioteca e da informação. Consiste em um esforço voluntário, com a finalidade de dispor de um recurso bibliográfico de acesso livre aos textos científicos especializados em ciências da informação.

- Eprints in Library and Information Science - E-LIS (//eprints.rclis.org): depósito de documentos especializados em biblioteconomia e ciências da informação. Promovido pelo Ministério Espanhol da Cultura e hospedado nos servidores do Consorzio Interuniversitario Lombardo per Elaborazione Automatica (CILEA). É o primeiro e-servidor temático internacional sobre documentação científica.

- Health InterNetwork Access to Research Initiative - HINARI (www.who.int/hinari/es): estabelecido pela OMS junto com as maiores casas editoriais, facilita o acesso a uma das mais extensas coleções de literatura biomédica e de saúde aos países em desenvolvimento. Mais de 3.070 revistas estão disponíveis para instituições de saúde em 113 países.

- Latindex (www.latindex.org): sistema regional de informação on line para revistas científicas da América Latina, Caribe, Espanha e Portugal. Conta com mais de 3.000 revistas da área das ciências médicas. É produto da cooperação de uma rede de instituições que funcionam de maneira coordenada para reunir e disseminar informação bibliográfica sobre as publicações científicas seriadas produzidas na região. 
- Los Alamos Preprint Archive - arXiv (arxiv.org): serviço de livre acesso e consulta nos seguintes campos: física, matemática, informática e biologia quantitativa. O conteúdo do arXiv está de acordo com os padrões acadêmicos da Universidade de Cornell, de Nova York. Propriedade, manutenção e financiamento por parte desta Universidade e co-financiado pela National Science Foundation.

- Online Access to Research in the Environment - OARE (www.oaresciences.org/es/): é uma aliança público-privada sob os auspícios do Programa das Nações Unidas para o Meio Ambiente (PNUMA), da Universidade de Yale e destacadas casas editoriais científicas e tecnológicas, que permite aos países em desenvolvimento acessarem, de forma gratuita, uma das coleções mais vastas de literatura sobre ciências ambientais do mundo.

- Open Access Repositories - OpenDOAR (www.opendoar.org/index.html): diretório de armazenamento acadêmico com acesso aberto. Cada depósito do OpenDOAR foi visitado pelo pessoal do projeto para comprovar a informação que se registra. É desenvolvido e mantido pela Universidade de Nottingham.

- Public Library of Science - PloS (www.plos.org/): organização não lucrativa composta por cientistas e médicos comprometidos em fazer da literatura científica e médica um recurso público. Recebeu ajuda financeira da Fundação de Gordon e de Betty Moore, da Fundación Sandler, da Fundação de Irving A. Hansen Memorial, do Open Society Institute (OSI) e do Joint Information Systems Committee (JISC). Também recebe doações e patrocínios de cidadãos particulares, de universidades e de outras organizações. PloS Medicine baseia seu sistema editorial na cobrança ao autor ou à instituição ao qual pertence.

- PubMed Central (www.pubmedcentral.nih.gov/): projeto desenvolvido e mantido pelo Centro Nacional para a Informação de Biotecnología (NCBI) da National Library of Medicine de Bethesda, nos Estados Unidos, que facilita o acesso livre e irrestrito ao material científico armazenado nessa plataforma digital. Proporciona acesso livre a mais de 227 revistas.

- Research Papers in Economics - RePEc (repec.org): ferramenta que resulta da colaboração de centenas de voluntários em 59 países, com a finalidade de realizar a difusão da pesquisa na economia. $\mathrm{O}$ fundamento do projeto é constituído por uma base de dados descentralizada dos trabalhos originais, artigos de revistas e de componentes informáticos (software). Todo o material da RePEc está livremente disponível de forma permanente e gratuita. Não contém artigos de texto completo, apenas proporciona vínculos (links) aos documentos acessíveis na rede por parte de outras instituições e de particulares.

- Scientific Electronic Library Online - SciELO (www. scielo.org/index.php?lang=es): obra do Centro Latinoamericano e do Caribe de Informação em Ciências da Saúde, da Organização Pan-americana de Saúde, Organização Mundial da Saúde (BIREME/OPS/OMS). O principal objetivo é o de contribuir para o desenvolvimento da pesquisa, aumentando a difusão da produção científica nacional, melhorando e ampliando os meios de publicação e avaliação de seus resultados.

- SciELO España (scielo.isciii.es/scielo.php/lng_es): desenvolvido pela Biblioteca Nacional de Ciências da Saúde do Instituto de Saúde Carlos III de Madri. O SciELO Espanha é uma biblioteca virtual formada por uma coleção de revistas científicas espanholas de ciências da saúde, selecionadas de acordo com certos critérios de qualidade preestabelecidos.

- The Scholarly Publishing and Academic Resources Coalition - SPARC (www.arl.org/sparc): aliança entre universidades, bibliotecas, pesquisa e organizações acadêmicas. A coalizão é uma iniciativa da Associação das Bibliotecas de Pesquisa Norte-americanas (Association of Research Libraries in North America - ARL). Foi criada em 1997 para ser uma resposta construtiva às disfunções do mercado no sistema de comunicação do âmbito acadêmico.

- SPARC Europe (www.sparceurope.org): cópia da Scholarly Publishing and Academic Resources Coalition (SPARC) no âmbito europeu. As universidades espanholas presentes são: Universidade de Las Palmas, da Grã Canária, e Universitat Politécnica de Catalunya.

\section{Reflexão final}

Vivemos em um mundo complexo, global e tecnicizado, no qual a informação ocupa um lugar fundamental para a tomada de decisões e para o desenvolvimento da ação no cotidiano. Portanto, existe o risco de que ocorra uma fratura entre setores, zonas, regiões e países quanto à capacidade de usar a informação.

Fala-se da ameaça da exclusão digital daqueles que não podem utilizar a revolução tecnológica. A iniciativa open access, o livre acesso à informação, deve exercer uma ação fundamental como veículo de suporte àqueles privados das vantagens do acesso à literatura científica.

A falta de igualdade de acesso à produção científica mundial permite uma reflexão final: enquanto uma parte do mundo pode aproximar-se da insanidade pelas várias formas de excessos nas práticas da pesquisa, a outra parte tratará de buscar formas de superação nem sempre satisfatórias para resolver essa brecha de informação, que, por sua vez, gera maior descompasso digital.

Se é possível afirmar que as novas tecnologias nos aproximam da informação, superando barreiras geográficas, também é certo admitir que geram novas necessidades. Hoje em dia, um pesquisador mal documentado e desconhecedor das vias pelas quais circulam os novos conhecimentos se converte em alguém "indocumentado", que seria sinônimo de pessoa não qualificada, despreparada para exercer seu trabalho, para atuar no mundo. Portanto, esta iniqüidade no acesso à literatura científica, que o movimento Open Access deve evitar, pode gerar uma nova forma de analfabetismo, se é que já não a concebeu!(SANZ-VALERO, 2006).

À guisa de conclusão, temos certeza de que o êxito da iniciativa Open Access se localiza no progressivo apoio da comunidade científica e de suas instituições. Almejamos que assim seja. 


\section{Referências bibliográficas}

ANDERSON, R. Author disincentives and open access. Serials Review, v.30, n.4, p.288-291, 2004.

ANTELMAN K. Do open access articles have a greater research impact? College \& Research Libraries, v.65, n.5, p.372-82, 2004.

BAILEY, C.W., Jr. Open access bibliography. Liberating Scholarly Literature with E-Prints and Open Access Journals. Washington: Association of Research Libraries; 2005. Disponível em: http://www.escholarlypub.com/oab/ oab.pdf. Acesso em: 23 feb 2007.

CANCELA RODRÍGUEZ, P. Manejo de la base de datos MEDLINE y otras aplicaciones de utilidad en Internet para la medicina oral. Profesión Dental, v.6, p.177-80, 2003.

CAÑEDO ANDALIA, R. Las revistas científicas actuales: la tormenta tras la calma. Acimed, v.10, n.5, 2002. [about 11 screens]. Disponível em:http://www. bvs.sld.cu/revistas/aci/voll0_5_02/aci06502.htm. Acesso em : 22 feb 2007.

EPRINTS.org. University of Southampton; c2002 Self Archiving FAQ; [about 37 screens]. Disponível em: http:// www.eprints.org/self-faq. Acesso em: 22 jan 2007.

HARNARD S. Post Gutenberg galaxy: the fourth revolution in the means of production of knowledge. PublicAccess Computer Systems Review, v.2, n.1, 1991. [about 19 screens]. Disponível em: http://epress.lib. uh.edu/pr/v2/nl/harnad.2nl. Acesso em: 20 feb 2007.

HORTON, R.; SMITH, R. Time to register randomised trials. British Medical Journal, v.319, n.865, p.6, 1999.

HARNARD, S.; BRODY, T. Comparing the Impact of Open Access (OA) vs. Non-OA Articles in the Same Journals. D-Lib Magazine, v.10, n.6, 2004. [about 8 screens]. Disponível em: http://www.dlib.org/dlib/june04/ harnad/06harnad.html.Acesso em: 20 feb. 2007.

HOORN, E, VAN DER GRAAF, M. Copyright Issues in Open Access Research Journals. D-Lib Magazine. v. 12, n.2, 2006 [about 14 screens]. Disponível em: http://www. dlib.org/dlib/february06/vandergraaf/02vandergraaf. html. Acesso em: 24 feb. 2006

JAÉN CASQUERO, B. Documentación a texto completo. En: BOJO CANALES, C, et al. Internet Visible e Invisible: búsqueda y selección de recursos de información en Ciencias de la Salud. Madrid: Instituto de Salud Carlos III, 2004. p. 65-78.

LIBRARIES take a stand: Journals present rising costs to libraries - and to scholarship; Harvard Gazette Archives. Cambridge (MA): The Harvard University; 2004. Disponível em: http://www.news.harvard.edu/gazette/2004/02.05/10-libraries.html.

LIMA LEITE, F.C.; MÁRDERO ARELLANO, M.A.; PASSINI MORENO. F. Acesso livre a publicações e repositórios digitais em ciência da informação no Brasil.
Perspectivas em Ciência da Informação, Belo Horizonte, v.11, n.1, p.82-94, 2006.

MELERO, R. Acceso abierto a las publicaciones científicas: definición, recursos, copyright e impacto. El Profesional de la Información, v.14, n.4, p.255-66, 2005.

MELERO, R.; PÉREZ-AGÜERA, J.R. Plataforma digital de revistas científicas electrónicas españolas. Relación con el movimiento open access. Scripta Nova, v.8, n.170, 2004. Disponível em: http://www.ub.es/geocrit/ sn/sn-170-74.htm. Acesso em:21 feb. 2007.

MUÑOZ TINOCO C. Iniciativas y tendencias de la edición electrónica de la literatura científica: acceso abierto. In: Bibliodoc; Anuari de biblioteconomia, documentació i informació. Barcelona: Col-legi Oficial de BibliotecarisDocumentalistes de Catalunya, 2005. p. 65-89.

OPCIT. The Open Citation Project. Southampton: Department of Electronics \& Computer Science, University of Southampton. Disponível em: http://opcit.eprints.org/ Acesso em: 26 feb. 2007

OPEN Access Charter. BioMed Central, London, 1999-2005. Disponível em: http://www.biomedcentral. com/info/about/charter. Acesso em: 22 jan 2007.

PERINE, L. La Internet: Plataforma en constante expansión para la investigación mundial. Inter-forum. Disponível em: http://www.revistainterforum.com/espanol/articulos/lperine artl.html. Acesso em: 22 jan. 2007.

REASONS to publish in BioMed Central's journals. BioMed Central, London,1999-2005. Disponível em http://www.biomedcentral.com/info/authors/reasons. Acesso em: 22.jan. 2007.

SANZ-VALERO, J. Do homo sapiens ao homo documentalis, passando pelo homo virtualis. In: CASTIEL, L.D.; VASCONCELLOS-SILVA, P.R. Precariedades dos excessos: informação e comunicação em saúde coletiva. Rio de Janeiro: Editora Fiocruz; 2006. p. 7-11.

SANZ-VALERO, J. et al. V. Internet y la búsqueda de información en Salud Pública: desde la relevancia hacia la "revelancia". Gaceta Sanitaria, v.20, n.2, p.159-60, 2006.

SCHROTER, S.; TITE, L.; SMITH, R. Perceptions of Open Access publishing: interviews with journal authors. Bristish Medical Journal, v.330, p.756-759, 2005.

SOCIEDAD MAX PLANCK. La declaración de Berlín sobre acceso abierto. GeoTrópico, v.1, n.2, 2003. Disponível em: http://www.geotropico.org/1_2_Documentos_Berlin.html. Acesso em: 22 feb. 2007.

SUBER, P. Timeline of the Free Online Scholarship Movement. Disponível em: http://www.earlham.edu/ $\sim$ peters/fos/timeline.htm. Acesso em: 23 feb. 2007.

THOMSON CORPORATION. The impact of open access journals: A Citation Study from Thomson ISI. New York: The Thomson Corporation; 2004. Disponível 
em: http://www.thomsonscientific.com/media/presentrep/ acropdf/impact-oa-journals.pdf

VASCONCELLOS-SILVA, P. R.; CASTIEL, L. D.; RIVERA, F. J. Evaluación mediante análisis de registros de un sitio de información de salud en Internet: la experiencia del Instituto Nacional del Cáncer de Brasil. Revista Panamericana de Salud Pública, v. 14, p.134137, 2003.

VAZQUEZ VALERO, M. et al. Visibilidad revistas científicas española. Madrid, 2003. Disponível em: http://wwwn.mec.es/univ/html/informes/estudios_analisis/resultados 2003/EA2003-0026/EA2003-0026.pdf. Acesso em: 02 feb 2007.
VEIGA DE CABO, J. La biblioteca virtual en salud (BVS): una apuesta por la difusión de la producción científica española y latinoamericana en colaboración con la OPS/ OMS. Revista Española de Salud Pública, v.75, n.4, p.277-80, 2001.

VEIGA DE CABO, J. Visibilidad de revistas científicas e iniciativas para incrementar la difusión de las publicaciones españolas. Nutrición Hospitalaria, v.18, n.4, p.177-80, 2003.

VIVES GRÀCIA, J. Aspectos de propiedad intelectual en la creación y gestión de repositorios institucionales. El Profesional de la Información, v.14, n.4, p.267278, 2005.

\section{Sobre os autores}

\section{Javier Sanz-Valero}

Doutor em Saúde Pública pela Universidad de Alicante (Espanha), Mestre em Saúde Pública e Gestão Sanitária pela Universidad de Valencia (Espanha), com diploma em Estudos Avançados de Documentação Científica e em Metodologia de Investigação em Ciências da Saúde. É pesquisador da linha de Comunicação e Documentação da Área de Nutrição do Centro Superior de Investigación en Salud Pública (CSISP), de Valência na Espanha. Também é professor assistente e pesquisador da linha de documentação científica da História da Ciência da Universidad de Alicante. Além disso, é membro dos grupos: Red de Malnutrición en Iberoamericana (Red Mel - CYTED); Comunicação e Documentação Científica aplicada à Nutrição da Sociedad Española de Nutrición Parenteral y Enteral (CDC-Nut SENPE); da junta diretiva da Sociedad Valenciana para el Estudio de la Calidad (SoVEC); do Comitê Científico da Revista Medicina y Seguridad del Trabajo e, assessor do Comitê Editorial da Revista Nutrición Hospitalaria.

\section{Jorge Veiga}

Graduado em Medicina pela Universidad de Salamanca na Espanha (1983), diplomado em Sanidad e mestrado em Pesquisa Clínica pela Escuela Nacional de Sanidad de Madrid (Espanha). Foi diretor da Biblioteca Nacional de Ciencias de la Salud da Espanha onde desenvolveu o Índice Bibliográfico Espanhol de Ciência e da Saúde e o Catálogo Coletico de Publicações Periódicas das Bibliotecas de Ciencias de la Salud Españolas, Biblioteca Virtual en Salud e Scientific Electronic Library Online (SciELO) Espanha, sendo os últimos em colaboração com a BIREME (OPS/OMS), instituição que continua a colaborar na qualidade de consultor. Atualmente é chefe do Serviço de Divulgação Científica da Escuela Nacional de Medicina del Trabajo, sendo responsável pelo Programa de Editorial e redator chefe da Revista de Medicina y Seguridad del Trabajo. É membro do grupo de Comunicação e Documentação Científica aplicada da Sociedad Española de Nutrición Parenteral (CDC-Nut SENPE) e do Centro Superior de Investigación en Salud Pública de Valencia. É também colaborador do projeto de terminologia Médica da Real Academia Española. 